\title{
DEGRADAÇÃO DE CORANTES ÁCIDOS POR PROCESSOS OXIDATIVOS AVANÇADOS USANDO UM REATOR COM DISCO ROTATÓRIO DE BAIXA VELOCIDADE
}

\author{
Vilson Bedim Vianna e Alexandre Rodrigues Tôrres
}

Departamento de Química e Ambiental, Faculdade de Tecnologia, Universidade do Estado do Rio de Janeiro, Rodovia Presidente Dutra, km 298, 27537-000 Resende - RJ, Brasil

Eduardo Bessa Azevedo*

Departamento de Química e Física Molecular, Instituto de Química de São Carlos, Universidade de São Paulo, CP 780, 13560-970

São Carlos - SP, Brasil

Recebido em 11/6/07; aceito em 27/3/08; publicado na web em 1/9/08

\begin{abstract}
ACID DYES DEGRADATION BY ADVANCED OXIDATION PROCESSES USING A LOW-SPEED ROTATING DISK REACTOR. Three technologies were tested $\left(\mathrm{TiO}_{2} / \mathrm{UV}, \mathrm{H}_{2} \mathrm{O}_{2} / \mathrm{UV}\right.$, and $\left.\mathrm{TiO}_{2} / \mathrm{H}_{2} \mathrm{O}_{2} / \mathrm{UV}\right)$ for the degradation and color removal of a $25 \mathrm{mg} \mathrm{L}^{-1} \mathrm{mixture}$ of three acid dyes: Blue 9, Red 18, and Yellow 23. A low speed rotating disc reactor $(20 \mathrm{rpm})$ and a $\mathrm{H}_{2} \mathrm{O}_{2}$ concentration of 2.5 mmol $\mathrm{L}^{-1}$ were used. The dyes did not significantly undergo photolysis, although they were all degraded by the studied advanced oxidation processes. With the $\mathrm{TiO}_{2} / \mathrm{H}_{2} \mathrm{O}_{2} / \mathrm{UV}$ process, a strong synergism was observed (color removal reached $100 \%$ ). Pseudo first order kinetic constants were estimated for all processes, as well as the respective apparent photonic efficiencies.
\end{abstract}

Keywords: dyes; $\mathrm{POA} ; \mathrm{TiO}_{2} ; \mathrm{H}_{2} \mathrm{O}_{2}$.

\section{INTRODUÇÃO}

O descarte de efluentes contendo altas concentrações de corantes é um problema bem conhecido associado a vários tipos de indústrias. Devido às taxas de fixação relativamente baixas, variando entre 60 e $90 \%^{1}$ e à baixa eficiência dos processos biológicos normalmente usados para o tratamento destes efluentes, ${ }^{2}$ cerca de $20 \%$ dos corantes não-fixados são descartados no meio ambiente. ${ }^{3}$ Torna-se, portanto, necessário investigar novas alternativas adequadas ao tratamento deste tipo de poluentes.

Os processos oxidativos avançados (POAs) têm sido extensivamente estudados, principalmente devido à sua capacidade de degradar um grande número de substâncias recalcitrantes através de procedimentos de custo relativamente baixo e de simples operação. Estes processos se baseiam na geração in situ do radical hidroxila $\left(\mathrm{OH}^{*}\right)$, cujo potencial padrão de oxidação só é superado pelo do flúor. Para uma série de compostos, a mineralização total tem sido alcançada.

Este trabalho teve por objetivo estudar a eficiência de três POAs $\left(\mathrm{H}_{2} \mathrm{O}_{2} / \mathrm{UV}, \mathrm{TiO}_{2} / \mathrm{UV}\right.$ e $\left.\mathrm{TiO}_{2} / \mathrm{H}_{2} \mathrm{O}_{2} / \mathrm{UV}\right)$ para a degradação de corantes ácidos (utilizados nas indústrias alimentícia, farmacêutica, de cosméticos e têxtil).

$\mathrm{O}$ processo $\mathrm{H}_{2} \mathrm{O}_{2} / \mathrm{UV}$ é baseado na clivagem homolítica do peróxido de hidrogênio quando ele é irradiado com luz ultravioleta $\operatorname{com} \lambda$ $\leq 254 \mathrm{~nm}$, conforme Equação 1 .

$\mathrm{H}_{2} \mathrm{O}_{2} \stackrel{h v}{\longrightarrow} 2 \mathrm{OH}^{\bullet}$

Na fotocatálise $\left(\mathrm{TiO}_{2} / \mathrm{UV}\right)$, luz ultravioleta (UV) $\operatorname{com} \lambda<380 \mathrm{~nm}$ $\mathrm{e}$, portanto, com energia superior à barreira entre a banda de valência e a banda de condução do $\mathrm{TiO}_{2}$, induz à formação de elétrons na banda de valência e de vacâncias positivas na banda de condução. ${ }^{4}$ Estas espécies carregadas podem se recombinar, liberando a energia absorvida como calor, ou podem migrar para a superfície da partícula

\footnotetext{
*e-mail: bessa@iqsc.usp.br
}

do fotocatalisador. Na presença da água ou do íon hidróxido, o radical hidroxila $\left(\mathrm{OH}^{\circ}\right)$ é formando na superfície do semicondutor. O processo é mostrado de forma simplificada pelas Equações 2-4.

$\mathrm{TiO}_{2} \stackrel{h \mathrm{v}}{\longrightarrow} \mathrm{h}_{\mathrm{BV}}{ }^{+}+\mathrm{e}_{\mathrm{BC}}{ }^{-}$

$\mathrm{h}_{\mathrm{BV}}{ }^{+}+\mathrm{H}_{2} \mathrm{O}($ ads $) \longrightarrow \mathrm{OH}^{\bullet}+\mathrm{H}^{+}$

$\mathrm{h}{ }^{+}+\mathrm{OH}^{-}(a d s) \longrightarrow \mathrm{OH}^{\bullet}$

Já o processo $\mathrm{TiO}_{2} / \mathrm{H}_{2} \mathrm{O}_{2} / \mathrm{UV}$ é empregado buscando-se um sinergismo que aumente a produção do $\mathrm{OH}^{*} \mathrm{e}$, conseqüentemente, as taxas de degradação.

Foram estudados três corantes ácidos que representam três classes químicas distintas: triarilmetanos, xantenos e azos. Suas principais características são mostradas na Tabela 1. Além disso, o azul brilhante (Acid Blue 9) tem sido associado a efeitos mutagênicos e bioacumulação, a eritrosina (Acid Red 18) a tumores cancerígenos e a tartrazina (Acid Yellow 23) a reações alérgicas. ${ }^{5-8}$

$\mathrm{O}$ reator utilizado neste estudo consiste de um disco que gira dentro de um reservatório contendo a espécie a ser degradada, sendo que, a cada momento, a metade inferior está imersa na solução e a metade superior está exposta ao ar. As lâmpadas ultravioletas são posicionadas de forma a irradiar a metade superior.

Este tipo de reator tem duas características importantes: primeiramente, não há necessidade de se oxigenar o meio, pois a própria difusão do oxigênio do ar para a superfície do catalisador é suficiente para manter processo oxidativo; em segundo lugar, há a formação de um filme delgado na metade superior do disco, facilitando sobremaneira a penetração dos fótons. Essa última característica é bastante vantajosa quando se lida com matrizes com forte coloração, como é o caso de efluentes contendo corantes, que podem acabar por agir como um filtro, evitando que os fótons cheguem ao fotocatalisador e, por conseqüência, a reação de degradação fotocatalítica.

Experimentos com esse reator têm sido relatados pelo grupo do 
Tabela 1. Principais características dos corantes ácidos estudados

\begin{tabular}{|c|c|c|c|c|c|}
\hline Nome & Color Index (C.I.) & Classe Química & Massa Molar $\left(\mathrm{g} \mathrm{mol}^{-1}\right)$ & Fórmula Estrutural & \\
\hline Acid Blue 9 & 42090 & Triarilmetano & 792,84 & & \\
\hline Acid Red 18 & 45430 & Xanteno & 879,87 & & \\
\hline Acid Yellow 23 & 19140 & Azo & 534,37 & & \\
\hline
\end{tabular}

Prof. D. D. Dionysiou da Universidade de Cincinnati, EUA. Esse grupo tem estudado a degradação de fenol, compostos fenólicos e pesticidas, sempre operando o reator em regime contínuo e no estado estacionário. Eles relatam degradações variando de 63\% (lindano) a $86 \%$ (ácido 4-cloro-benzóico), nas condições por eles testadas. Também relatam aumentos de aproximadamente duas vezes nas taxas de degradação ao se usar o processo acoplado $\left(\mathrm{TiO}_{2} / \mathrm{H}_{2} \mathrm{O}_{2} / \mathrm{UV}\right)$. No presente trabalho, esse reator foi usado para a degradação de corantes, sendo operado no modo batelada.

\section{PARTE EXPERIMENTAL}

\section{Reagentes}

Os corantes foram fornecidos pela Empresa Supreme Resources, sendo utilizados nas suas formas originais. O fotocatalisador utilizado foi o $\mathrm{TiO}_{2}$ P25 da Degussa (tamanho de partícula de $30 \mathrm{~nm}$, área BET de $50 \pm 15 \mathrm{~m}^{2} \mathrm{~g}^{-1}$ e composição de $70 \%$ de anatásio e $30 \%$ de rutilo). ${ }^{9}$ Todos os demais reagentes utilizados foram de grau PA e usados como recebidos.

\section{Reator}

Foi utilizado um reator com disco rotatório de baixa velocidade adaptado, ${ }^{10,11}$ conforme mostrado na Figura 1. O disco do reator foi confeccionado em alumínio. Em ambas as faces do disco de alumínio

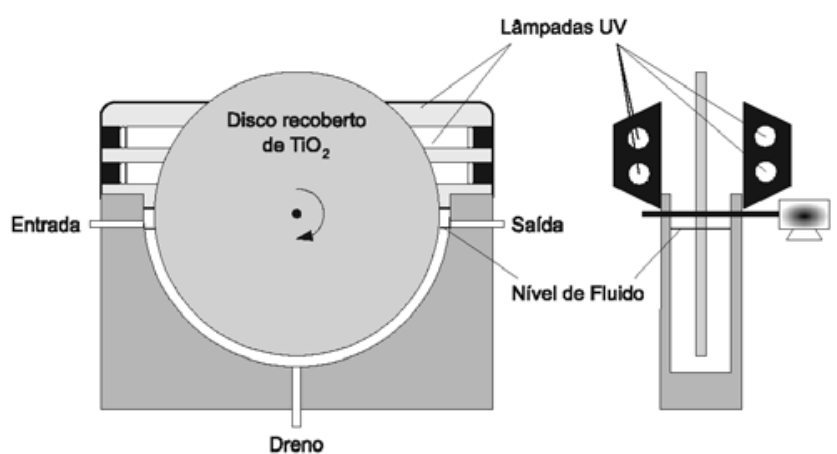

VISTA FRONTAL (EM CORTE)

VISTA LATERAL foram colados discos de vidro jateado. Este conjunto foi utilizado para o tratamento com $\mathrm{H}_{2} \mathrm{O}_{2} / \mathrm{UV}$. Nos casos dos tratamentos com $\mathrm{TiO}_{2} / \mathrm{UV}$ e $\mathrm{TiO}_{2} / \mathrm{H}_{2} \mathrm{O}_{2} / \mathrm{UV}$, usou-se um outro conjunto no qual o fotocatalisador foi imobilizado ${ }^{12}$ nos discos de vidro jateado. Os discos foram previamente lavados com detergente e ácido nítrico diluído (10\%). Em seguida, foram feitas sucessivas aplicações (10) de uma suspensão a $1 \%$ de $\mathrm{TiO}_{2}$ em pH 3 (ajustado com ácido nítrico). Após cada aplicação, a solução foi parcialmente escoada e a placa foi seca com ar quente. Por fim, os discos foram mantidos por $1 \mathrm{~h}$ em estufa à $100^{\circ} \mathrm{C}$ para garantir melhor fixação do dióxido de titânio.

$O$ reator foi operado a uma velocidade angular de $20 \mathrm{rpm}^{13,14}$ (velocidade angular na qual não há limitações à transferência de massa, ou seja, o reator encontra-se perfeitamente misturado) e o volume útil do reator era de 4,5 L. Foram utilizadas 4 lâmpadas germicidas de 15 W Light Express modelo LE15W (emissão principal em $254 \mathrm{~nm}$ ). A distribuição da intensidade luminosa no disco foi determinada com a ajuda de um radiômetro da marca Cole Parmer Instruments Co., modelo EW-09811-54, com sensor para leitura no comprimento de onda de $254 \mathrm{~nm}$. A Figura 2 apresenta a distribuição obtida.

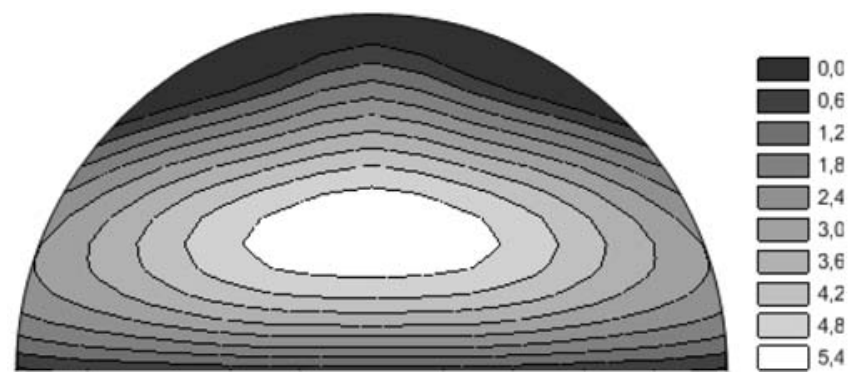

Figura 2. Distribuição da intensidade luminosa (I) no disco rotatório. Escala: valores de I em $\mathrm{mW} \mathrm{cm}^{-2}$

\section{Experimentos}

A solução testada continha $25 \mathrm{mg} \mathrm{L}^{-1}$ de cada corante dissolvido em água destilada. Nos tratamentos que usaram o peróxido de hidrogênio, a concentração de $\mathrm{H}_{2} \mathrm{O}_{2}$ foi fixada em $2,5 \mathrm{mmol} \mathrm{L}^{-1}$ (concentrações baseadas em estudos prévios). ${ }^{15}$

O tempo máximo de reação foi de $360 \mathrm{~min}$, tendo sido coletadas amostras a cada 15 min na $1^{\text {a }}$ hora e a cada 30 min até o término do experimento. Todos os experimentos foram feitos em triplicata.

Figura 1. Esquema do reator fotocatalítico 


\section{Análises}

Em cada amostra retirada nos tempos pré-definidos, realizouse uma varredura em um espectrofotômetro Shimadzu UV-160A na região do UV/Visível (200 a $750 \mathrm{~nm}$ ), obtendo-se, assim, os respectivos espectros. Em seguida, foram estimadas as constantes cinéticas e determinadas as respectivas significâncias estatísticas usando-se uma rotina de minimização dos quadrados dos desvios entre os pontos experimentais e os estimados. Além disso, integrouse a área sob os espectros na região visível (400 a $750 \mathrm{~nm}$ ) e estes valores de área foram utilizados como uma medida do descoramento da solução.

\section{RESULTADOS E DISCUSSÃO}

Inicialmente, foram obtidos os espectros na região do UV-Visível de cada corante separadamente. $O$ resultado obtido é mostrado na Figura 3. Os máximos de absorção observados foram: Blue 9 (629,5 $\mathrm{nm})$, Red 18 (526,0 nm) e Yellow 23 (427,5 nm).

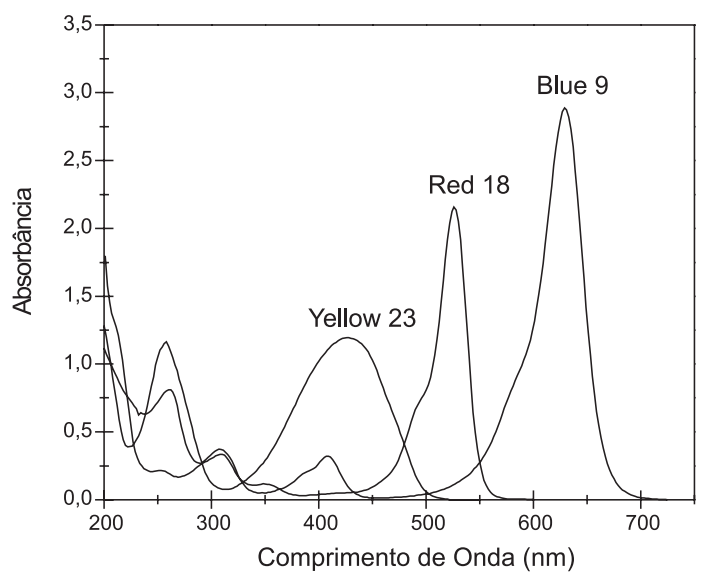

Figura 3. Espectros na região do UV-Visível dos corantes ácidos utilizados

Em seguida, foram calculadas as absortividades dos corantes nos três respectivos máximos: Blue $9\left(0,13 \mathrm{~L} \mathrm{mg}^{-1} \mathrm{~cm}^{-1}\right)$, Red 18 $\left(0,094 \mathrm{~L} \mathrm{mg}^{-1} \mathrm{~cm}^{-1}\right)$ e Yellow $23\left(0,047 \mathrm{~L} \mathrm{mg}^{-1} \mathrm{~cm}^{-1}\right)$. De posse das absortividades, foi então possível calcular, a partir dos espectros obtidos das amostras retiradas do reator, a concentração de cada um dos corantes ao longo da reação.

\section{Fotólise}

Os resultados obtidos para a fotólise mostraram que não houve degradação significativa da mistura, já que os espectros na região UV-Visível (200 a $750 \mathrm{~nm}$ ) obtidos foram praticamente coincidentes.

A observação anterior é corroborada quando são calculadas as concentrações residuais dos corantes em cada tempo de irradiação. Ao final de $6 \mathrm{~h}$, o Blue 9 e o Yellow 23 praticamente não sofreram fotólise (1,4 e 0,5\% de degradação, respectivamente). Já o Red 18, embora tenha sofrido fotólise, esta não foi muito significativa, atingindo apenas 9,5\% de degradação.

Dois modelos foram aplicados para a estimativa das constantes cinéticas de fotólise do Red 18: um de ordem zero e outro de pseudo $1^{\mathrm{a}}$ ordem. Os valores obtidos foram $(1,6 \pm 0,15) \times 10^{-2}$ $\mathrm{mg} \mathrm{L}^{-1} \mathrm{~h}^{-1}\left(\mathrm{R}^{2}=0,993\right)$ e $(1,7 \pm 0,13) \times 10^{-2} \mathrm{~h}^{-1}\left(\mathrm{R}^{2}=0,995\right)$, respectivamente, para um coeficiente de determinação máximo de 0,996 . Verifica-se que ambos podem ser usados satisfatoriamente para descrever o processo.

\section{Espectros no UV-Visível obtidos durante a degradação dos} corantes

A Figura 4 apresenta uma amostra dos espectros na região do UV-Visível obtidos para cada tipo de tecnologia empregada.

Durante a degradação dos corantes, aparentemente não foi formada nenhuma substância (intermediário, produto final) que absorvesse na região espectral analisada. Isto pode ser constatado pela diminuição monotônica dos espectros.

Os três corantes são passíveis de degradação pelas tecnologias testadas. Isto é uma das características mais importantes dos POAs, ou seja, são tecnologias inespecíficas, sendo capazes de destruir praticamente qualquer tipo de molécula ou grupamentos.

Embora tenha sido o único a apresentar uma fotólise apreciável, o corante que apresenta a menor taxa de reação é o Red 18. De fato, com $6 \mathrm{~h}$ de irradiação, as amostras finais submetidas aos processos $\mathrm{TiO}_{2} / \mathrm{UV}$ e $\mathrm{H}_{2} \mathrm{O}_{2} / \mathrm{UV}$ permaneceram levemente avermelhadas. Entretanto, com o processo combinado $\mathrm{TiO}_{2} / \mathrm{H}_{2} \mathrm{O}_{2} / \mathrm{UV}$, a solução inicial foi totalmente descorada.

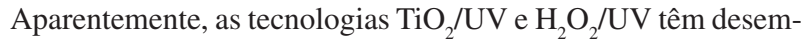
penhos semelhantes. Já o acoplamento das duas aumentou significativamente a taxa de degradação da mistura de corantes.
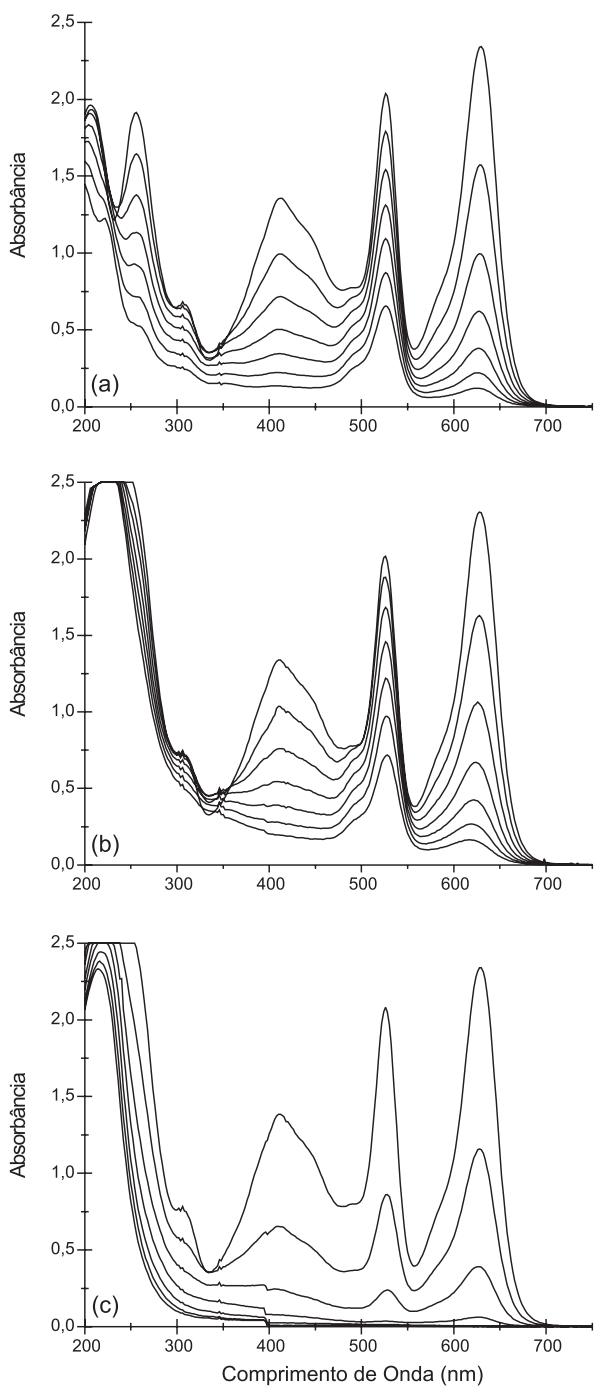

Figura 4. Espectros obtidos pela varredura no UV-Visível da mistura inicial e após 1, 2, 3, 4, 5 e 6 h de irradiação: (a) $\mathrm{TiO}_{2} / U V$, (b) $\mathrm{H}_{2} \mathrm{O}_{2} / U V$ e (c) $\mathrm{TiO}_{2} / \mathrm{H}_{2} \mathrm{O}_{2} / \mathrm{UV}$ 
O aumento de absorção na região entre 200 e 330 nm nas Figuras $4 \mathrm{~b}$ e $4 \mathrm{c}$ deve-se à presença do peróxido de hidrogênio.

\section{Degradação dos corantes}

A Figura 5 apresenta os resultados médios alcançados para a degradação dos três corantes testados com cada tecnologia. Verifica-se que o comportamento das duas tecnologias separadamente é, de fato, bastante semelhante, enquanto aquele do acoplamento das duas é bem diferente, tendo ocorrido um aumento significativo na degradação dos três corantes. Para as tecnologias $\mathrm{TiO}_{2} / \mathrm{UV} \mathrm{e}_{2} \mathrm{O}_{2} / \mathrm{UV}$, respectivamente, foram obtidas as seguintes degradações após $6 \mathrm{~h}$ de irradiação: Blue 9 - 93 e 95\%; Red $18-73$ e $61 \%$ e Yellow $23-92$ e $86 \%$. Para o processo combinado, as degradações foram de 100, 100 e $98 \%$ para os corantes Blue 9, Red 18 e Yellow 23, respectivamente.

Entretanto, um fato se manteve constante: a degradação do Yellow 23 , independentemente da tecnologia empregada, foi sempre menor que a do Blue 9 .

A Figura 6 mostra o comportamento do descoramento da mistura em função do processo oxidativo utilizado. A região sombreada é o intervalo de predição estimado. Novamente, percebe-se que o acoplamento das duas tecnologias aumenta o descoramento de forma sensível.
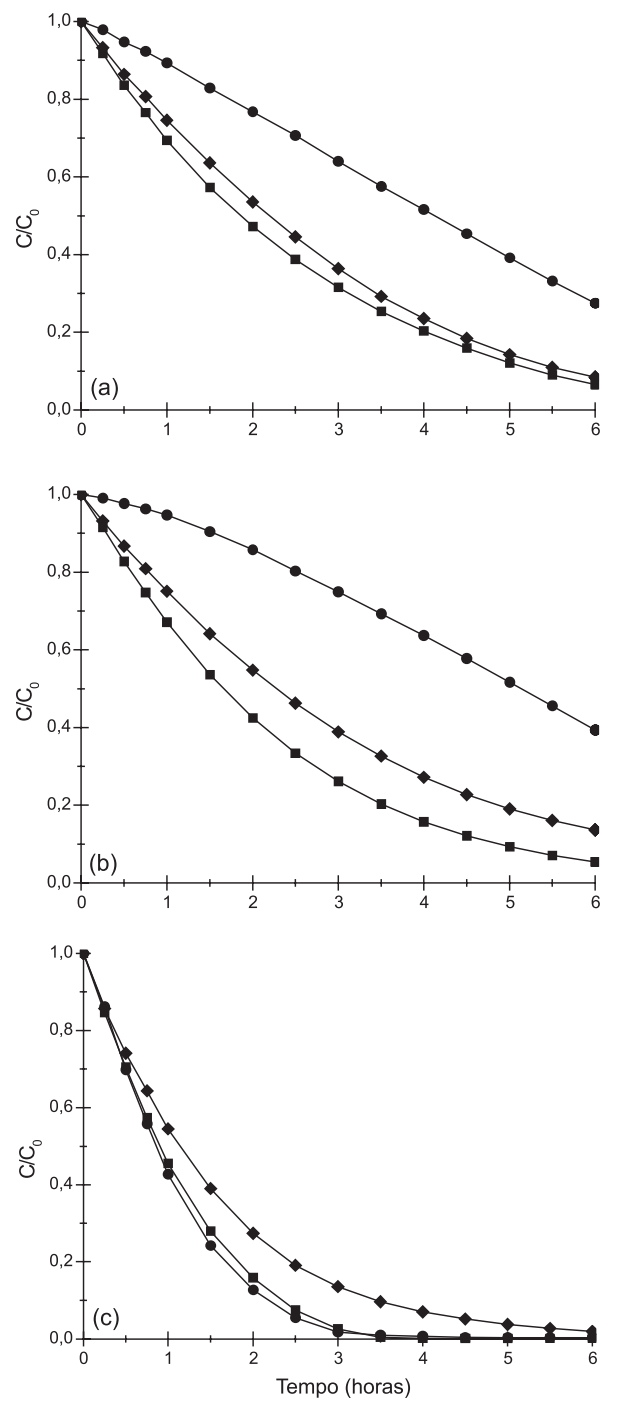

Figura 5. Cinética de degradação dos corantes ácidos Blue 9 (---), Red 18

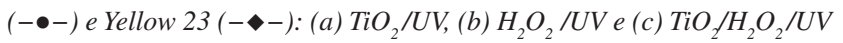
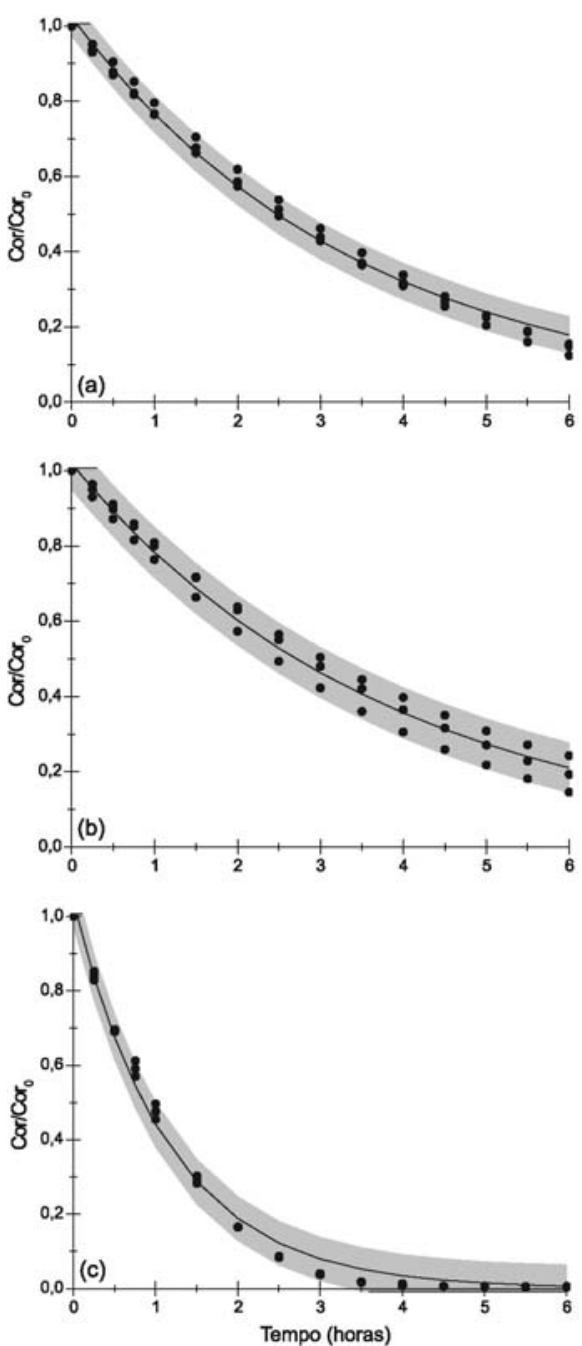

Figura 6. Cinética (pseudo $1^{a}$ ordem) de descoramento: (a) $\mathrm{TiO}_{2} / \mathrm{UV}$, (b) $\mathrm{H}_{2} \mathrm{O}_{2} / \mathrm{UVe}$ (c) $\mathrm{TiO}_{2} / \mathrm{H}_{2} \mathrm{O}_{2} / \mathrm{UV}$. Os círculos são os pontos experimentais, as linhas o comportamento estimado e a área sombreada, o intervalo de predição

A Tabela 2 resume, para os três processos estudados $\left(\mathrm{TiO}_{2} / \mathrm{UV}\right.$, $\mathrm{H}_{2} \mathrm{O}_{2} / \mathrm{UV}$ e $\mathrm{TiO}_{2} / \mathrm{H}_{2} \mathrm{O}_{2} / \mathrm{UV}$ ), as constantes cinéticas estimadas (pseudo $1^{\mathrm{a}}$ ordem) de degradação para cada corante e a de descoramento, além dos respectivos coeficientes de determinação e do coeficiente de determinação máximo que poderia ser obtido a partir dos dados experimentais disponíveis. Em muitos estudos de degradação fotocatalítica com o $\mathrm{TiO}_{2}$, devido às baixas concentrações iniciais dos poluentes, o uso do modelo de pseudo $1^{\mathrm{a}}$ ordem tem se mostrado bastante razoável. ${ }^{16}$

No caso do processo $\mathrm{TiO}_{2} / \mathrm{UV}$, verifica-se que as constantes do Blue 9 e do Yellow 23 são semelhantes, embora estatisticamente diferentes. Outra consideração relevante é que o modelo de pseudo $1^{\mathrm{a}}$ ordem se ajustou bem aos dados de degradação desses dois corantes, o que não ocorreu com os dados do Red 18. De fato, examinando-se a Figura 5a, percebe-se que o perfil de degradação é retilíneo e não exponencial. Ajustando-se o modelo de ordem zero aos dados do Red 18, obteve-se: $k=(1,2 \pm 0,12)$ $\times 10^{-1} \mathrm{mg} \mathrm{L}^{-1} \mathrm{~h}^{-1}\left(\mathrm{R}^{2}=0,990\right)$, que é o melhor ajuste possível aos dados experimentais. Ainda em relação ao Red 18 , comparando-se a constante cinética obtida com a dos demais corantes, verifica-se que ele é degradado aproximadamente duas vezes mais lentamente. Como era de se esperar, a constante cinética do descoramento apresentou um valor médio entre aqueles obtidos para os corantes.

As mesmas observações feitas anteriormente aplicam-se ao 


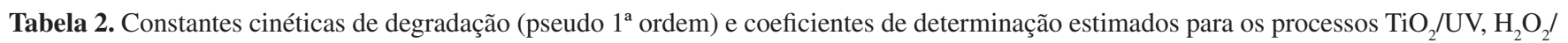
$\mathrm{UV} \mathrm{e} \mathrm{TiO}_{2} / \mathrm{H}_{2} \mathrm{O}_{2} / \mathrm{UV}$

\begin{tabular}{|c|c|c|c|c|c|c|}
\hline \multirow{2}{*}{$\begin{array}{c}\text { Processos } \\
\text { Parâmetros }\end{array}$} & \multicolumn{2}{|c|}{$\mathrm{TiO}_{2} / \mathrm{UV}$} & \multicolumn{2}{|c|}{$\mathrm{H}_{2} \mathrm{O}_{2} / \mathrm{UV}$} & \multicolumn{2}{|c|}{$\mathrm{TiO}_{2} / \mathrm{H}_{2} \mathrm{O}_{2} / \mathrm{UV}$} \\
\hline & $k, \mathrm{~h}^{-1}$ & $\mathrm{R}^{2}\left(\mathrm{R}^{2}\right.$ máx.) & $k, \mathrm{~h}^{-1}$ & $\mathrm{R}^{2}$ ( $\mathrm{R}^{2}$ máx.) & $k, \mathrm{~h}^{-1}$ & $\mathrm{R}^{2}$ ( $\mathrm{R}^{2}$ máx. $)$ \\
\hline Blue 9 & $(4,0 \pm 0,16) \times 10^{-1}$ & $\begin{array}{c}0,991 \\
(0,993)\end{array}$ & $(4,5 \pm 0,19) \times 10^{-1}$ & $\begin{array}{c}0,991 \\
(0,993)\end{array}$ & $(8,9 \pm 0,49) \times 10^{-1}$ & $\begin{array}{c}0,991 \\
(0,999)\end{array}$ \\
\hline Red 18 & $(1,9 \pm 0,12) \times 10^{-1}$ & $\begin{array}{c}0,968 \\
(0,990)\end{array}$ & $(1,4 \pm 0,13) \times 10^{-1}$ & $\begin{array}{c}0,926 \\
(0,964)\end{array}$ & $(9,5 \pm 0,57) \times 10^{-1}$ & $\begin{array}{c}0,989 \\
(0,997)\end{array}$ \\
\hline Yellow 23 & $(3,6 \pm 0,15) \times 10^{-1}$ & $\begin{array}{c}0,991 \\
(0,997)\end{array}$ & $(3,2 \pm 0,12) \times 10^{-1}$ & $\begin{array}{c}0,991 \\
(0,993)\end{array}$ & $(8,3 \pm 0,31) \times 10^{-1}$ & $\begin{array}{c}0,996 \\
(0,999)\end{array}$ \\
\hline Descoramento & $(2,9 \pm 0,091) \times 10^{-1}$ & $\begin{array}{c}0,994 \\
(0,994) \\
\end{array}$ & $(2,6 \pm 0,12) \times 10^{-1}$ & $\begin{array}{c}0,986 \\
(0,998) \\
\end{array}$ & $(8,6 \pm 0,41) \times 10^{-1}$ & $\begin{array}{l}0,999 \\
(1,00) \\
\end{array}$ \\
\hline
\end{tabular}

processo $\mathrm{H}_{2} \mathrm{O}_{2} / \mathrm{UV}$. Mais uma vez, o modelo de ordem zero ajustou -se melhor aos dados do Red 18, fornecendo uma constante cinética igual a $k=(1,0 \pm 0,21) \times 10^{-1} \mathrm{mg} \mathrm{L}^{-1} \mathrm{~h}^{-1}\left(\mathrm{R}^{2}=0,955\right)$.

Interessante notar que as constantes cinéticas obtidas para o Red 18 , com as tecnologias $\mathrm{TiO}_{2} / \mathrm{UV} \mathrm{e} \mathrm{H}_{2} \mathrm{O}_{2} / \mathrm{UV}$, independentemente do modelo ajustado, são uma ordem de grandeza maiores que aquelas obtidas unicamente com a fotólise, ficando claro então o papel do radical $\mathrm{OH}^{*}$.

No caso do acoplamento dos dois processos o resultado foi bem distinto. Pode-se observar que esta tecnologia funcionou nivelando as taxas de degradação, independentemente do tipo de corante. Em outras palavras, tornou o processo oxidativo ainda mais inespecífico. De fato, observando-se a Tabela 2 , verifica-se que as constantes de pseudo $1^{\mathrm{a}}$ ordem não são estatisticamente diferentes. Outra observação importante é que a degradação do Red 18 passou a ser razoavelmente bem descrita por um modelo de $1^{\mathrm{a}}$ ordem.

Vale a pena ainda frisar que o acoplamento das duas tecnologias produziu um sinergismo que aumentou, novamente, em uma ordem de grandeza as taxas de degradação dos corantes e de descoramento. Este sinergismo já foi relatado na literatura para um sistema equivalente ao utilizado, no qual foi estudada a degradação do ácido 4-cloro-benzóico. ${ }^{17}$

\section{Cálculo da eficiência fotônica aparente}

O rendimento quântico $(\phi)$, representado pelo número de moléculas transformadas em função do número de fótons absorvidos pelo $\mathrm{TiO}_{2}$, não foi calculado devido à dificuldade de se determinar o quanto da radiação foi espalhada pelo fotocatalisador. No seu lugar, foi cal-

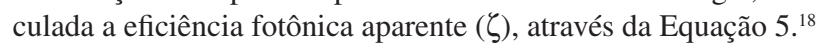

$$
\zeta=\frac{\text { taxa de transformação das moléculas }\left(\mathrm{mol} \mathrm{s}^{-1}\right)}{\text { taxa de incidência de fótons no disco }\left(\text { einstein } \mathrm{s}^{-1}\right)}
$$

A eficiência fotônica aparente é menor que o rendimento quântico, uma vez que leva em consideração a radiação espalhada pelo fotocatalisador (valores de rendimento quântico entre 1 e $5 \%$ são comuns). ${ }^{19,20}$ A energia de um mol de fótons (um quanta) de luz com $\lambda=254 \mathrm{~nm}$ foi calculada pela Lei da Radiação de Planck, ${ }^{21}$ conforme mostrado na Equação 6.

$$
E=\frac{1,19626 \times 10^{5}}{254}=471 \mathrm{~kJ} \mathrm{einstein}^{-1}
$$

A quantidade total de energia luminosa incidente no disco foi calculada pela integração da matriz de pontos obtida a partir das medidas realizadas com o radiômetro, utilizando-se o método RenkaCline e o programa OriginPro 7.5. O valor encontrado foi de 4.648 $\mathrm{mW}$, o que corresponde a $9,868 \times 10^{-5}$ einstein $\mathrm{s}^{-1}$.

A Tabela 3 mostra os valores calculados da eficiência fotônica aparente para cada corante e cada tecnologia empregada (levando-se em consideração a taxa inicial de remoção). Como era de se esperar, os valores são baixos em comparação com os de rendimento quântico. No entanto, fazendo-se as necessárias correções devido às diferentes massas molares, as eficiências calculadas concordam com dados da literatura. ${ }^{10}$

Tabela 3. Eficiências fotônicas aparentes (\%)

\begin{tabular}{cccc}
\hline \multirow{2}{*}{ Corantes } & \multicolumn{3}{c}{ Tecnologias } \\
& $\mathrm{TiO}_{2} / \mathrm{UV}$ & $\mathrm{H}_{2} \mathrm{O}_{2} / \mathrm{UV}$ & $\mathrm{TiO}_{2} / \mathrm{H}_{2} \mathrm{O}_{2} / \mathrm{UV}$ \\
\hline Blue 9 & 0,130 & 0,136 & 0,244 \\
Red 18 & 0,0302 & 0,0130 & 0,199 \\
Yellow 23 & 0,160 & 0,160 & 0,339 \\
\hline
\end{tabular}

Analisando-se a Tabela 3, observa-se que o acoplamento das duas tecnologias $\left(\mathrm{TiO}_{2} / \mathrm{H}_{2} \mathrm{O}_{2} / \mathrm{UV}\right)$ aproximadamente dobrou a eficiência fotônica aparente no caso dos corantes ácidos Blue 9 e Yellow 23, enquanto a aumentou em uma ordem de grandeza no caso do corante Red 18. Os baixos valores encontrados são devidos certamente ao espalhamento da radiação pelo fotocatalisador e, talvez, signifiquem que também havia um excesso de fótons no sistema. Estudos posteriores nos quais a intensidade luminosa será variada poderão esclarecer melhor a contribuição desses dois processos.

\section{CONCLUSÕES}

Os corantes ácidos estudados, com a configuração de reator e com as lâmpadas utilizadas como fonte de luz ultravioleta, não sofreram fotólise significativa. No entanto, eles se mostraram suscetíveis de serem degradados pelos processos oxidativos avançados empregados, independentemente de apresentarem grupos cromóforos e estruturas diferentes. Esta é uma das características mais marcantes e desejáveis dos POAs, ou seja, sua inespecificidade.

Os processos $\mathrm{TiO}_{2} / \mathrm{UV}$ e $\mathrm{H}_{2} \mathrm{O}_{2} / \mathrm{UV}$ apresentaram desempenho semelhante, tanto em relação à degradação dos corantes quanto ao descoramento da mistura. Neles, a ordem de degradação observada foi: Blue $9>$ Yellow $23>>$ Red 18 .

Já o processo acoplado $\mathrm{TiO} / \mathrm{H}_{2} \mathrm{O}_{2} / \mathrm{UV}$ apresentou um forte sinergismo, tendo sido alcançadas remoções próximas a $100 \%$. As taxas de degradação dos corantes em média dobraram e a de descoramento da mistura triplicou. O processo mostrou-se ainda mais inespecífico, uma vez que as taxas de degradação não são estatisticamente diferentes. Além disso, o sinergismo também pôde ser observado através da eficiência fotônica aparente calculada, a qual dobrou no caso do Blue 9 e do Yellow 23 e aumentou uma ordem de grandeza no caso do Red 18. Essas observações são provavelmente devidas ao aumento na concentração dos radicais $\mathrm{OH}^{*}$, devido à clivagem homolítica do peróxido de hidrogênio. 
De maneira geral, o modelo cinético de pseudo $1^{\mathrm{a}}$ ordem se ajusta bem aos dados experimentais obtidos, com exceção do Red 18 que apresentou uma degradação de ordem zero quando foram utilizados os dois processos separadamente.

Por fim, pôde-se verificar que o reator com disco rotatório de baixa velocidade é adequado para a degradação dos corantes utilizados. As altas degradações obtidas podem ser atribuídas ao fato de se irradiar um filme delgado da solução dos poluentes, garantindo a chegada de uma quantidade suficiente de fótons à superfície do fotocatalisador.

\section{AGRADECIMENTOS}

Às empresas Supreme Resources e Degussa pelos corantes e pelo $\mathrm{TiO}_{2} \mathrm{P} 25$, respectivamente.

\section{REFERÊNCIAS}

1. Camp, R.; Sturrock, P. E.; Water Res. 1990, 24, 1275.

2. Gouvêa, C. A. K.; Wypych, F.; Moraes, S. G.; Duran, N.; Nagata, N.; Peralta-Zamoura, P.; Chemosphere 2000, 40, 433.

3. O'Neill, C.; Hawkes, F. R.; Hawkes, D. L.; Lourenço, N. D.; Pinheiro, H. M.; Delee, W.; J. Chem. Technol. Biotechnol. 1999, 74, 1009.

4. Linsebigler, A. L.; Lu, G.; Yates Jr., J. T.; Chem. Rev. 1995, 95, 735.

5. Aziz, A. H. A.; Shouman, S. A.; Attia, A. S.; Saad, S. F.; Pharm. Res. 1997, 35, 457.
6. Groten, J. P.; Butler, W.; Feron, V. J.; Kozianowski, G.; Renwick, A. C.; Walker, R.; Regulatory Toxicology and Pharmacology 2000, 31, 77.

7. Hess, E. V.; Toxicology 2002, 181-182, 65.

8. Alsolaiman, M. M.; Howard, L.; Nutrition 2003, 19, 395.

9. Degussa Technical Bulletin Pigments; Highly dispersed metallic oxides produced by the AEROSIL ${ }^{\circledR}$ process, 56, 1990.

10. Dionysiou, D. D.; Khodadoust, A. P.; Kern, A. M.; Suidan, M. T.; Baudin, I.; Laîne, J. -M.; Appl. Catal., B 2000, 24, 139.

11. Dionysiou, D. D.; Balasubramanian, G.; Suidan, M. T.; Khodadoust, A. P.; Baudin, I.; Laîné, J. -M.; Water Res. 2000, 34, 2927.

12. Nogueira, R. F. P.; Jardim, W. F.; Solar Energy 1996, 56, 471.

13. Dionysiou, D. D.; Suidan, M. T.; Baudin, I.; Laîne, J. -M. ; Appl. Catal., B 2002, 38, 1 .

14. Dionysiou, D. D.; Burbano, A. A.; Suidan, M. T.; Baudin, I.; Laîne, J. -M.; Environ. Sci. Technol. 2002, 36, 3834.

15. Figueiredo, A. M.; Andrade, P. R.; Azevedo, E. B.; Resumos da $13^{a}$ Semana de Iniciação Científica/UERJ, Rio de Janeiro, Brasil, 2004.

16. Al-Ekabi, H. ; Serpone, N.; J. Phys. Chem. 1988, 92, 264.

17. Dionysiou, D. D.; Suidan, M. T.; Baudin, I.; Laîné, J. -M.; Appl. Catal., B 2004, 50, 259.

18. Serpone N.; Journal of Advanced Oxidation Technologies 1997, 2, 203.

19. Legrini, O.; Oliveros, E.; Braun, A. M.; Chem. Rev. 1993, 93, 671.

20. Mills, G.; Hoffmann, M. R.; Environ. Sci. Technol. 1993, 27, 1681.

21. Stefan, M. I. Em Advanced Oxidation Processes for Water and Wastewater Treatment; Parsons, S., ed.; IWA Publishing: Londres, 2004, cap. 1. 